\title{
Propagation of a relativistic electron wave packet in the Dirac equation
}

\author{
Sang Tae Park ${ }^{*}$ \\ Arthur Amos Noyes Laboratory of Chemical Physics, California Institute of Technology, Pasadena, California 91125, USA
}

(Received 10 September 2012; published 12 December 2012)

\begin{abstract}
Solving the Dirac equation is a formidable task due to the high frequency and the degrees of freedom involved. However, this high frequency allows one to obtain an approximation to the equation. Here, we directly solve the Dirac equation using an envelope method and derive analytical solutions of Dirac wave packets to first order for the small momentum spread. We apply the insight gained from this solution to the Zitterbewegung behavior in a Dirac-like system, where we show that it is crucial to include the first-order term in our solution to correctly describe a Dirac packet.
\end{abstract}

DOI: 10.1103/PhysRevA.86.062105

PACS number(s): 03.65.Pm, 03.30.+p, 04.25.-g, 41.75.Ht

\section{INTRODUCTION}

The Dirac equation [1,2] successfully merges quantum mechanics with special relativity. Furthermore, it naturally describes the electron spin and predicts the existence of antimatter [3]. However, the Dirac equation also predicts some peculiar effects, such as Klein's paradox [4] and Zitterbewegung [5]. Schrödinger noticed that if a mathematical superposition state of positive and negative energies were evolved in time according to the single-particle Dirac equation, then the time-dependent expectation value of the position operator in this mathematical state reveals oscillatory motion with a speed of light, a period of $\frac{\hbar}{m c^{2}}$, and an amplitude proportional to the reduced Compton wavelength $\left(\frac{\hbar}{m c}\right)$ as a result of the interference between positive- and negativeenergy states. The Zitterbewegung is often presented as if it refers to an observable trembling motion associated with a real physical particle [6,7]; for example, it was suggested as the origin of the electron spin [8], and it is given as a reasoning for the Darwin term [9] in atomic fine structure. However, this concept is controversial and was challenged by Krekora et al. [10]. The existence of Zitterbewegung, in relativistic quantum mechanics and in quantum field theory, has been a recurrent subject of discussion over the past years [10-12]. The interest in Zitterbewegung was recently rekindled due to its analog in Dirac-like systems [13-18]. This is, in particular, because the length $\left(10^{-13} \mathrm{~m}\right)$ and time $\left(10^{-21} \mathrm{~s}\right)$ scales involved in Zitterbewegung are extremely small and short, beyond the current capability of detection, and a Dirac-like system allows one to study an analog of the Dirac equation in measurable length and time scales [13].

The position operator and its time evolution have been one of the main tools in investigating the Zitterbewegung [8,19,20]. A numerical approach recently has gained in popularity [13,21-25]. However, neither approach provides a physical insight on the mechanism of the phenomenon. The formulation is too abstract to deduce the mechanism in the operator method, whereas the intrinsic mechanism in lost in the numerical method. Interestingly, the Zitterbewegung term in the expectation value of the position operator vanishes on taking expectation values for wave packets that are made up entirely of positive- or negative-energy waves using a Foldy-Wouthuysen

\footnotetext{
*stpark@caltech.edu
}

transformation [26-29]. Similarly, in numerical simulations, it is only seen in position expectation values when positive- and negative-energy packets are overlapped. Therefore, Zitterbewegung is now understood to be an interference effect between the positive- and negative-energy parts of the Dirac solution. However, its physical origin is not yet fully understood.

This is all because there is no known analytical expression for a Dirac packet. In a previous publication [30], we obtained an analytical solution to a first-order approximation of the Dirac equation for an electron under an electromagnetic wave using envelope functions. Here, we utilize this approach to obtain an exact analytical solution to a first-order approximation of the Dirac equation for a relativistic freeelectron packet, which we employ to investigate the relativistic effects of a Dirac wave packet, in particular, Zitterbewegung, in the framework of the Dirac equation, a single-particle formalism. The position expectation value of the total wave function of a hypothetical superposition state is analytically derived to obtain the Zitterbewegung behavior. The nature of a superposition state and the Zitterbewegung in the Dirac equation and in the Dirac-like system is discussed using the analytical solution. Also discussed is the validity of the spinor wave packets widely used in numerical simulations.

\section{THEORY}

\section{A. Nonrelativistic Schrödinger wave packet}

The time-dependent Schrödinger equation for a free electron is given as

$$
i \hbar \frac{\partial \Psi}{\partial t}=-\frac{\hbar^{2}}{2 m} \nabla^{2} \Psi .
$$

We define a one-dimensional (1D) wave packet in terms of the envelope function $f(z, t)$ multiplied by the carrier wave, such that

$$
\Psi(z, t)=f(z, t) \exp \left[i\left(k_{c} z-\omega_{c} t\right)\right],
$$

where $\hbar k_{c}=m v_{0}$ and $\hbar \omega_{c}=\frac{1}{2} m v_{0}^{2}$, and we get

$$
\frac{\partial f}{\partial t}+\frac{\hbar k_{c}}{m} \frac{\partial f}{\partial z}-\frac{i \hbar}{2 m} \frac{\partial^{2} f}{\partial z^{2}}=0 .
$$

The second term is the propagation, with a velocity of $v_{0}=\frac{\hbar k_{c}}{m}$, and the third term is the dispersion. Equation (3) has an exact 
solution for a Gaussian packet:

$$
f(z, t)=\sqrt{\frac{1}{\sqrt{2 \pi}\left(\sigma_{z}+\frac{i \hbar t}{2 m \sigma_{z}}\right)} \exp \left[-\frac{\left(z-v_{0} t\right)^{2}}{2 \sigma_{z}\left(\sigma_{z}+\frac{i \hbar t}{2 m \sigma_{z}}\right)}\right]} .
$$

Then the wave function is retrieved by Eq. (2), and the probability density becomes

$$
P(z, t)=\frac{1}{\sqrt{2 \pi} \Sigma_{z}} \exp \left[-\frac{\left(z-v_{0} t\right)^{2}}{2 \Sigma_{z}^{2}}\right],
$$

where $\Sigma_{z}\left(t ; \sigma_{z}\right)=\sqrt{\sigma_{z}^{2}+d^{2}}$ and $d\left(t ; \sigma_{z}\right)=\frac{\hbar t}{2 m \sigma_{z}}$. We note that classically $d=\frac{\hbar \sigma_{k}}{m} t=\frac{\sigma_{p}}{m} t=\sigma_{v} t$ becomes a dispersion because for a Gaussian packet, $\sigma_{z}=\frac{1}{2 \sigma_{k}}$, as the wave functions in momentum and position spaces satisfy $\mathcal{F}^{-1}\{\Psi(k, 0)\}=$ $\Psi(z, 0)$ when

$$
\begin{gathered}
\Psi(k, 0)=\sqrt{\hat{G}\left(k-k_{c} ; \sigma_{k}\right),} \\
\Psi(z, 0)=\sqrt{G\left(z ; \sigma_{z}\right)} e^{i k_{c} z}
\end{gathered}
$$

at $t=0$, where $\hat{G}$ and $G$ are Gaussian profiles in momentum and position spaces, respectively,

$$
\begin{aligned}
& \hat{G}\left(k ; \sigma_{k}\right)=\frac{1}{\sqrt{2 \pi} \sigma_{k}} \exp \left[-\frac{(k)^{2}}{2 \sigma_{k}^{2}}\right], \\
& G\left(z ; \sigma_{z}\right)=\frac{1}{\sqrt{2 \pi} \sigma_{z}} \exp \left[-\frac{(z)^{2}}{2 \sigma_{z}^{2}}\right] .
\end{aligned}
$$

\section{B. Relativistic Dirac wave packet}

It is beneficial to recall relativistic relations of

$$
\gamma=\frac{1}{\sqrt{1-\left(\frac{v}{c}\right)^{2}}}=\sqrt{1+\left(\frac{p}{m c}\right)^{2}}=\frac{E}{m c^{2}},
$$

where $\gamma$ is the relativistic factor, $m$ is the mass at rest, $c$ is the speed of light, $v$ is the velocity, $p$ is the momentum, and $E=T+m c^{2}$ is the relativistic energy. The Dirac equation for a free electron is given as

$$
i \hbar \frac{\partial \Psi}{\partial t}=\left\{c \boldsymbol{\alpha} \cdot \vec{p}+\boldsymbol{\beta} m c^{2}\right\} \Psi,
$$

where $\boldsymbol{\alpha}$ and $\boldsymbol{\beta}$ are unit constant matrices chosen to satisfy the relativistic energy-momentum relation. For one dimension in the $z$ direction, $\boldsymbol{\alpha}, \boldsymbol{\beta}$, and $\Psi$ are given by two components as

$$
\begin{gathered}
\boldsymbol{\alpha}=\left(\begin{array}{ll}
0 & 1 \\
1 & 0
\end{array}\right), \\
\boldsymbol{\beta}=\left(\begin{array}{cc}
1 & 0 \\
0 & -1
\end{array}\right), \\
\Psi=\left(\begin{array}{l}
\psi_{1} \\
\psi_{2}
\end{array}\right) .
\end{gathered}
$$

The planar wave solutions with momentum $p$ are given as

$$
\Psi^{+}=\left(\begin{array}{c}
u_{1}^{+} \\
u_{2}^{+}
\end{array}\right) \exp \left[i\left(k z-\omega^{+} t\right)\right]
$$

$$
\Psi^{-}=\left(\begin{array}{l}
u_{1}^{-} \\
u_{2}^{-}
\end{array}\right) \exp \left[i\left(k z-\omega^{-} t\right)\right]
$$

where

$$
\begin{gathered}
\hbar k=p, \\
\hbar \omega^{+}=E^{+}=+\sqrt{p^{2} c^{2}+m^{2} c^{4}}, \\
\hbar \omega^{-}=E^{-}=-\sqrt{p^{2} c^{2}+m^{2} c^{4}}, \\
+u_{1}^{+}=\frac{E^{+}+m c^{2}}{\sqrt{2 E^{+}\left(E^{+}+m c^{2}\right)}}=+\sqrt{\frac{\gamma+1}{2 \gamma}}, \\
+u_{2}^{+}=\frac{p c}{\sqrt{2 E^{+}\left(E^{+}+m c^{2}\right)}}= \pm \sqrt{\frac{\gamma-1}{2 \gamma}}, \\
-u_{1}^{-}=\frac{p c}{\sqrt{2 E^{-}\left(E^{-}-m c^{2}\right)}}= \pm \sqrt{\frac{\gamma-1}{2 \gamma}}, \\
-u_{2}^{-}=\frac{E^{-}-m c^{2}}{\sqrt{2 E^{-}\left(E^{-}-m c^{2}\right)}}=-\sqrt{\frac{\gamma+1}{2 \gamma}},
\end{gathered}
$$

where + and - on the right-hand side refer to when $p>0$ and $p<0$, respectively. The positive-energy solution corresponds to the matter, electron, and the negative-energy solution corresponds to the antimatter, positron. Here, spinor coefficients $u_{i}^{ \pm}$are expressed more conveniently with $\gamma$ using Eq. (10). Figure 1 shows the $p$ dependence of spinor vectors. Note that the positive- and the negative-energy spinor vectors are orthogonal at any given momentum.

Similar to Eq. (6), a Gaussian wave packet for an electron in the Dirac equation can be constructed by superposition of a spectrum of positive-energy planar wave solutions [31,32] in momentum space,

$$
\Psi_{e}(k, 0)=\sqrt{\hat{G}\left(k-k_{0} ; \sigma_{k}\right)}\left(\begin{array}{c}
u_{1}^{+}(k) \\
u_{2}^{+}(k)
\end{array}\right),
$$

where the subscript 0 designates the values at the center of the distribution. However, spinor coefficients $u_{1}^{+}$and $u_{2}^{+}$ also depend on the momentum (see Fig. 1), and their $k$ dependence needs to be explicitly considered (see Sec. S.I A in the Supplemental Material [33]). Spinor coefficients can be Taylor expanded as

$$
u_{i}^{ \pm}(k)=\left.\sum_{n=0}^{\infty} \frac{\left(k-k_{0}\right)^{n}}{n !} \frac{d^{n} u_{i}^{ \pm}}{d k^{n}}\right|_{k=k_{0}} .
$$

It can be shown that, for the first-order derivative,

$$
\begin{aligned}
& \frac{d u_{1}^{+}}{d k}=\mp \frac{1}{2 \gamma^{2}} \sqrt{\frac{\gamma-1}{2 \gamma}}\left(\frac{\hbar}{m c}\right)=+\frac{\lambda_{C}}{2 \gamma^{2}} u_{1}^{-}, \\
& \frac{d u_{2}^{+}}{d k}=+\frac{1}{2 \gamma^{2}} \sqrt{\frac{\gamma+1}{2 \gamma}}\left(\frac{\hbar}{m c}\right)=+\frac{\lambda_{C}}{2 \gamma^{2}} u_{2}^{-},
\end{aligned}
$$

and

$$
\begin{aligned}
& \frac{d u_{1}^{-}}{d k}=-\frac{1}{2 \gamma^{2}} \sqrt{\frac{\gamma+1}{2 \gamma}}\left(\frac{\hbar}{m c}\right)=-\frac{\lambda_{C}}{2 \gamma^{2}} u_{1}^{+}, \\
& \frac{d u_{2}^{-}}{d k}=\mp \frac{1}{2 \gamma^{2}} \sqrt{\frac{\gamma-1}{2 \gamma}}\left(\frac{\hbar}{m c}\right)=-\frac{\lambda_{C}}{2 \gamma^{2}} u_{2}^{+},
\end{aligned}
$$




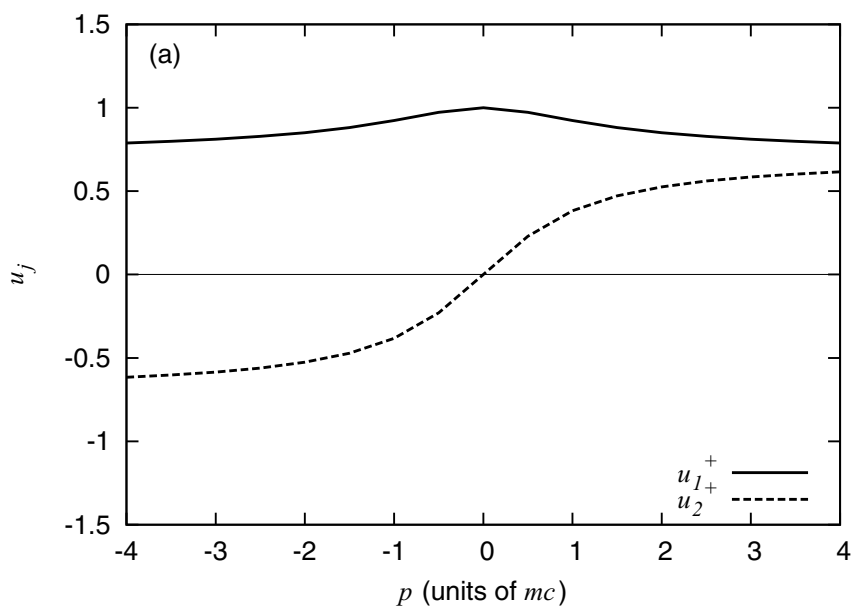

(b)

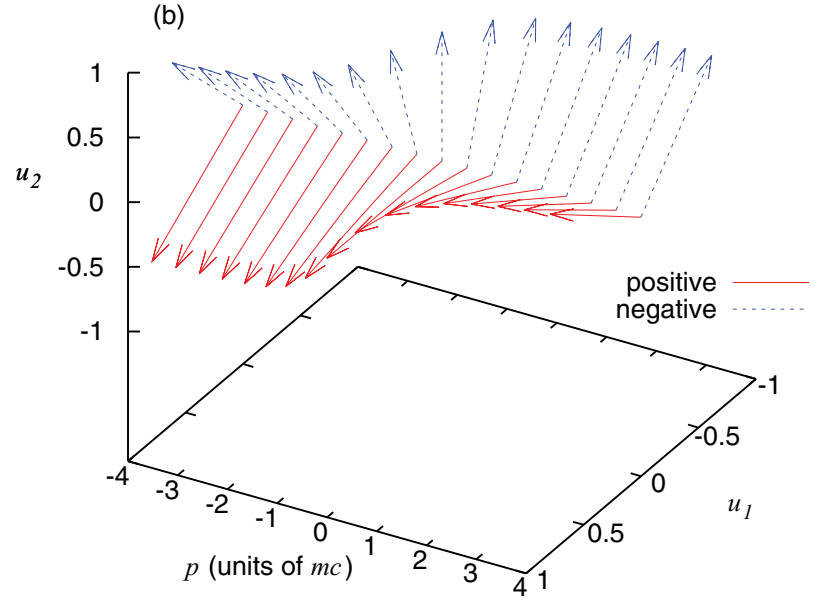

FIG. 1. (Color online) (a) Dependences of positive-energy Dirac spinor coefficients on the momentum. (b) Three-dimensional visualization of spinor vectors of positive and negative energies as a function of momentum.

where $\lambda_{C}=\left(\frac{\hbar}{m c}\right)=3.86 \times 10^{-13} \mathrm{~m}$ is the reduced Compton wavelength. For convenience, we designate spinor vectors

$$
\hat{\boldsymbol{u}}_{(k)}^{ \pm} \equiv\left(\begin{array}{c}
u_{1}^{ \pm}(k) \\
u_{2}^{ \pm}(k)
\end{array}\right)
$$

such that

$$
\frac{d}{d k} \hat{\boldsymbol{u}}_{(k)}^{ \pm}= \pm \frac{\lambda_{C}}{2 \gamma^{2}} \hat{\boldsymbol{u}}_{(k)}^{\mp} .
$$

Using $\mathcal{F}^{-1}\left\{i^{n} k^{n} \hat{F}(k)\right\}=\left(\frac{d}{d z}\right)^{n} \mathcal{F}^{-1}\{\hat{F}(k)\}$, we get

$$
\begin{aligned}
\Psi_{e}(k, 0)= & \sum_{n=0}^{\infty} \frac{1}{n !}\left\{\left(\frac{d}{d k}\right)^{n} \hat{\boldsymbol{u}}_{(k)}^{+}\right\}_{k=k_{0}}\left(k-k_{0}\right)^{n} \\
& \times \sqrt{\hat{G}\left(k-k_{0} ; \sigma_{k}\right)} \\
= & \left\{\hat{\boldsymbol{u}}_{\left(k_{0}\right)}^{+}+\left(\frac{\lambda_{C}}{2 \gamma_{0}^{2}}\right) \hat{\boldsymbol{u}}_{\left(k_{0}\right)}^{-}\left(k-k_{0}\right)-\cdots\right\} \\
& \times \sqrt{\hat{G}\left(k-k_{0} ; \sigma_{k}\right)},
\end{aligned}
$$

$$
\begin{aligned}
\Psi_{e}(z, 0)= & \exp \left[i k_{0} z\right]\left\{\hat{\boldsymbol{u}}_{\left(k_{0}\right)}^{+} \sqrt{G\left(z ; \sigma_{z}\right)}-i \hat{\boldsymbol{u}}_{\left(k_{0}\right)}^{-}\left(\frac{\lambda_{C}}{2 \gamma_{0}^{2}}\right)\right. \\
& \left.\times \frac{d \sqrt{G\left(z ; \sigma_{z}\right)}}{d z}+\cdots\right\} .
\end{aligned}
$$

Equation (34) shows that when represented in position space, the electron wave packet contains a small imaginary contribution with the negative-energy spinor vector, which appears as a positron-like component. It will be shown later that this positron-like component is, in fact, a part of the electron wave packet and should not be ignored. It is most convenient to express the wave function with the positive- and the negative-energy-component envelope functions as

$$
\Psi(z, t)=f^{+}(z, t) \hat{\boldsymbol{u}}_{\left(k_{0}\right)}^{+} e^{i\left(k_{0} z-\omega_{0}^{+} t\right)}+f^{-}(z, t) \hat{\boldsymbol{u}}_{\left(k_{0}\right)}^{-} e^{i\left(k_{0} z-\omega_{0}^{-} t\right)} .
$$

The evolution of the wave packet is then described by $f^{+}(z, t)$ and $f^{-}(z, t)$, which can be solved using Eq. (11). By substituting Eq. (35) in Eq. (11) and eliminating quantities that satisfy the relativistic energy-momentum relations of the carrier wave, we get a Dirac equation in terms of envelope functions (for $k_{0}>0$ ) as

$$
\begin{aligned}
& \frac{\partial f^{+}}{\partial c t}+\frac{\sqrt{\gamma_{0}^{2}-1}}{\gamma_{0}} \frac{\partial f^{+}}{\partial z}+\frac{1}{\gamma_{0}} \frac{\partial f^{-}}{\partial z} e^{i\left(\omega_{0}^{+}-\omega_{0}^{-}\right) t}=0, \\
& \frac{\partial f^{-}}{\partial c t}-\frac{\sqrt{\gamma_{0}^{2}-1}}{\gamma_{0}} \frac{\partial f^{-}}{\partial z}+\frac{1}{\gamma_{0}} \frac{\partial f^{+}}{\partial z} e^{i\left(\omega_{0}^{-}-\omega_{0}^{+}\right) t}=0 .
\end{aligned}
$$

The second terms on the left sides of Eqs. (36) and (37) represent the propagations of $f^{+}(z, t)$ and $f^{-}(z, t)$ with velocities of $v^{+}=+c \frac{\sqrt{\gamma_{0}^{2}-1}}{\gamma_{0}}=+v_{0}$ and $v^{-}=-c \frac{\sqrt{\gamma_{0}^{2}-1}}{\gamma_{0}}=$ $-v_{0}$, respectively. Note that Eqs. (36) and (37) are coupled differential equations, and there is no apparent dispersion term. It will be shown that the dispersion arises from the coupled terms.

Equations (36) and (37) are not directly solvable (to our best knowledge). However, a close inspection reveals that a certain approximation is possible as follows: Eq. (37) has two contributions, one from the minor component, $f^{-}$, and the other from the major component, $f^{+}$, when solving for an electron. Since $f^{-}(z, t)$ propagates in the opposite direction away from $f^{+}(z, t)$, it does not cumulate, and the only contribution is from the portion where the initial wave packet is very small and from $f^{+}(z, t)$. An approximate solution for $f^{-}(z, t)$ in Eq. (37) can be obtained [see Eq. (A1)] by assuming that the temporal behavior of $f^{-}(z, t)$ is dominated by the fast oscillation terms $e^{i\left(\omega_{0}^{-}-\omega_{0}^{+}\right) t}$ as

$$
f^{-}(z, t) \approx-\frac{1}{i\left(\omega_{0}^{-}-\omega_{0}^{+}\right)} \frac{c}{\gamma_{0}}\left(\frac{\partial f^{+}}{\partial z}\right) e^{i\left(\omega_{0}^{-}-\omega_{0}^{+}\right) t},
$$

which approximately satisfies the major component part, $\frac{\partial f^{-}}{\partial t}+\frac{c}{\gamma_{0}}\left(\frac{\partial f^{+}}{\partial z}\right) e^{i\left(\omega_{0}^{-}-\omega_{0}^{+}\right) t} \approx 0$, for very large $\pm \omega_{0}^{ \pm}$and relatively slowly varying $f^{+}(z, t)$ (high-frequency approximation). An equivalent approximation was obtained in a small kinetic energy limit (nonrelativistic approximation) [34]. However, we recognize that the validity of our approximation lies in the limit of a small momentum distribution width $\sigma_{k}$, not the absolute value of the momentum $\hbar k_{0}$ or the kinetic energy 
$\hbar \omega_{0}^{+}-m c^{2}$. It should also be noted that Eq. (38) agrees with Eq. (34) at $t=0$ because $\hbar \omega_{0}^{ \pm}= \pm \gamma_{0} m c^{2}$. Using Eq. (38), we get an uncoupled differential equation for an electron with $f^{+}(z, t)$ only from Eq. (36) as

$$
\frac{\partial f^{+}}{\partial t}+v_{0} \frac{\partial f^{+}}{\partial z}-\frac{i \hbar}{2 \gamma_{0}^{3} m} \frac{\partial^{2} f^{+}}{\partial z^{2}} \approx 0,
$$

which is equivalent to Eq. (3), the nonrelativistic counterpart, except for a relativistic correction, $\gamma_{0}^{3}$, to the mass in the dispersion term. This effective mass is known as the relativistic longitudinal mass, $m_{L}=\gamma^{3} m=\frac{F}{a}=\frac{\partial p}{\partial v}$.

Equation (39) has an exact solution for a Gaussian packet:

$$
f_{e}^{+}(z, t)=\sqrt{\frac{1}{\sqrt{2 \pi}\left(\sigma_{z}+\frac{i \hbar t}{2 \gamma_{0}^{3} m \sigma_{z}}\right)} \exp \left[-\frac{\left(z-v_{0} t\right)^{2}}{2 \sigma_{z}\left(\sigma_{z}+\frac{i \hbar t}{2 \gamma_{0}^{3} m \sigma_{z}}\right)}\right]},
$$

$$
f_{e}^{-}(z, t)=-\frac{i \hbar}{2 \gamma_{0}^{2} m c}\left(\frac{\partial f_{e}^{+}}{\partial z}\right) \exp \left[i\left(\omega_{0}^{-}-\omega_{0}^{+}\right) t\right]
$$

which is valid for $\sigma_{z} \gg \lambda_{C}$, or $2 \sigma_{p} \ll m c$, and the wave function becomes

$$
\begin{aligned}
\Psi_{e}(z, t)= & C_{e}\left\{f_{e}^{+}(z, t) \hat{\boldsymbol{u}}_{\left(k_{0}\right)}^{+}-i \frac{\lambda_{C}}{2 \gamma_{0}^{2}}\left(\frac{\partial f_{e}^{+}(z, t)}{\partial z}\right) \hat{\boldsymbol{u}}_{\left(k_{0}\right)}^{-}\right\} \\
& \times \exp \left[i\left(k_{0} z-\omega_{0}^{+} t\right)\right],
\end{aligned}
$$

where $C_{e}=\left\{1+\frac{1}{4 \sigma_{z}^{2}}\left(\frac{\lambda_{C}}{2 \gamma_{0}^{2}}\right)^{2}\right\}^{-\frac{1}{2}}$ is the normalization correction due to truncation in Eq. (34). The probability density becomes

$$
\begin{aligned}
P_{e}(z, t)= & \frac{C_{e}^{2}}{\sqrt{2 \pi} \Sigma_{z}} \exp \left[-\frac{\left(z-v_{0} t\right)^{2}}{2 \Sigma_{z}^{2}}\right] \\
& \times\left[1+\left(\frac{\lambda_{C}}{2 \gamma_{0}^{2}}\right)^{2} \frac{1}{2 \sigma_{z}^{2}} \frac{\left(z-v_{0} t\right)^{2}}{2 \Sigma_{z}^{2}}\right],
\end{aligned}
$$

where $\Sigma_{z}\left(t ; \sigma_{z}\right)=\sqrt{\sigma_{z}^{2}+d^{2}}$ and $d\left(t ; \sigma_{z}\right)=\frac{\hbar t}{2 \gamma_{0}^{3} m \sigma_{z}}=\frac{\hbar \sigma_{k} t}{\gamma_{0}^{3} m}=$ $\frac{\sigma_{p} t}{\gamma_{0}^{3} m}$. We note that in the relativistic relation $\sigma_{p} \approx \gamma_{0}^{3} m \sigma_{v}$ from $\frac{\partial p}{\partial v}=\gamma^{3} m$, and therefore $d \approx \sigma_{v} t$, which is equivalent to the nonrelativistic counterpart. Equation (43) results in $\langle z\rangle=+v_{0} t$, which is equivalent to classical mechanics. We note that the single-particle wave packet for an electron does not show any trembling motion.

Similarly, the uncoupled differential equation for a positron with $f^{-}(z, t)$ only becomes

$$
\frac{\partial f^{-}}{\partial t}-v_{0} \frac{\partial f^{-}}{\partial z}+\frac{i \hbar}{2 \gamma_{0}^{3} m} \frac{\partial^{2} f^{-}}{\partial z^{2}} \approx 0,
$$

and the positron-only solution becomes

$$
f_{p}^{-}(z, t)=\sqrt{\frac{1}{\sqrt{2 \pi}\left(\sigma_{z}-\frac{i \hbar t}{2 \gamma_{0}^{3} m \sigma_{z}}\right)} \exp \left[-\frac{\left(z+v_{0} t\right)^{2}}{2 \sigma_{z}\left(\sigma_{z}-\frac{i \hbar t}{2 \gamma_{0}^{3} m \sigma_{z}}\right)}\right]},
$$

$$
f_{p}^{+}(z, t)=+\frac{i \hbar}{2 \gamma_{0}^{2} m c}\left(\frac{\partial f_{p}^{-}}{\partial z}\right) \exp \left[i\left(\omega_{0}^{+}-\omega_{0}^{-}\right) t\right],
$$

and the wave function becomes

$$
\begin{aligned}
\Psi_{p}(z, t)= & C_{p}\left\{f_{p}^{-}(z, t) \hat{\boldsymbol{u}}_{\left(k_{0}\right)}^{-}+i \frac{\lambda_{c}}{2 \gamma_{0}^{2}}\left(\frac{\partial f_{p}^{-}(z, t)}{\partial z}\right) \hat{\boldsymbol{u}}_{\left(k_{0}\right)}^{+}\right\} \\
& \times \exp \left[i\left(k_{0} z-\omega_{0}^{-} t\right)\right],
\end{aligned}
$$

where $\quad C_{p}=\left\{1+\frac{1}{4 \sigma_{z}^{2}}\left(\frac{\lambda_{C}}{2 \gamma_{0}^{2}}\right)^{2}\right\}^{-\frac{1}{2}}$ is the normalization correction. $\Psi_{e}(z, t)$ and $\Psi_{p}(z, t)$ are orthogonal as $\int_{-\infty}^{+\infty} d z \Psi_{p}^{\dagger}(z, t) \Psi_{e}(z, t)=0$ for symmetric Gaussian packets. It should be mentioned that the above equations hold true even for particles at rest, where $k_{0}=0, v_{0}=0$, and $\gamma_{0}=1$.

\section{RESULTS}

\section{A. Validation of analytical solutions: Zitterbewegung behavior}

In the previous section, we derived analytical solutions for a single-particle wave-packet propagation in the Dirac equation and showed that they do not exhibit any trembling motion [see Eq. (43)]. Nevertheless, Eq. (42) is only a first-order approximation, the validity of which needs to be verified. Since our solutions also apply to a Dirac-like system [13-18] which is a Schrödinger equation with an interaction Hamiltonian in a two-level system and therefore equivalent to the Dirac equation, we shall investigate the Zitterbewegung behavior of a superposition state in Dirac-like systems to validate those analytical solutions. Readers are referred to Sec. IV C for discussions on a distinction between a Dirac system and a Dirac-like system.

Let us assume a hypothetical situation of a superposition state where two wave packets for the positive-energy component and the negative-energy component overlap at $z=0$ and $t=0$. In Dirac-like systems, they correspond to the upper and the lower states, respectively, whereas in the Dirac equation they would correspond to the electron and the positron. Superposition coefficients are chosen to be real as

$$
\Psi(z, t)=\Psi_{e}(z, t) \cos \Theta+\Psi_{p}(z, t) \sin \Theta .
$$

Then the position expectation value of the total mixed wave function becomes [see Eq. (B1)]

$$
\begin{aligned}
\langle z\rangle \equiv & \left\langle\Psi^{\dagger}|z| \Psi\right\rangle \\
= & v_{0} t \cos 2 \Theta+\left\{1+\frac{1}{4 \sigma_{z}^{2}}\left(\frac{\lambda_{C}}{2 \gamma_{0}^{2}}\right)^{2}\right\}^{-1} \frac{\lambda_{C}}{2 \gamma_{0}^{2}} \sqrt{\frac{\sigma_{z}}{\Sigma_{z}}} \\
& \times \exp \left[-\frac{v_{0}^{2} t^{2}}{2 \Sigma_{z}^{2}}\right] \sin \left[2 \omega_{0} t-\frac{d^{2}}{\Sigma_{z}^{2}}\left(\gamma_{0}^{2}-1\right) \omega_{0} t+\frac{\phi}{2}\right] \\
& \times \sin 2 \Theta,
\end{aligned}
$$

where $2 \omega_{0}=\omega_{0}^{+}-\omega_{0}^{-}$and $\phi=\tan ^{-1}\left(\frac{d}{\sigma_{z}}\right)$. Equation (50) is valid when Eqs. (42) and (47) are valid. Figure 2 plots Eq. (50) for $p_{0}=0.967 m c$ and $2 \sigma_{p}=0.25 m c$ for $\Theta=\pi / 4$. The position expectation value of a mixed wave function shows an interference during their overlap. It arises from the fact that $\int_{-\infty}^{+\infty} d z \Psi_{p}(z, t)^{\dagger} z \Psi_{e}(z, t) \neq 0$ even though $\Psi_{e}(z, t)$ and $\Psi_{p}(z, t)$ are orthogonal (see Sec. II B). In Eq. (50), the (1D) interference pattern is sinusoidal, with a maximum amplitude of

$$
A_{Z B}=\left\{1+\frac{1}{4 \sigma_{z}^{2}}\left(\frac{\lambda_{C}}{2 \gamma_{0}^{2}}\right)^{2}\right\}^{-1} \frac{\lambda_{C}}{2 \gamma_{0}^{2}} .
$$



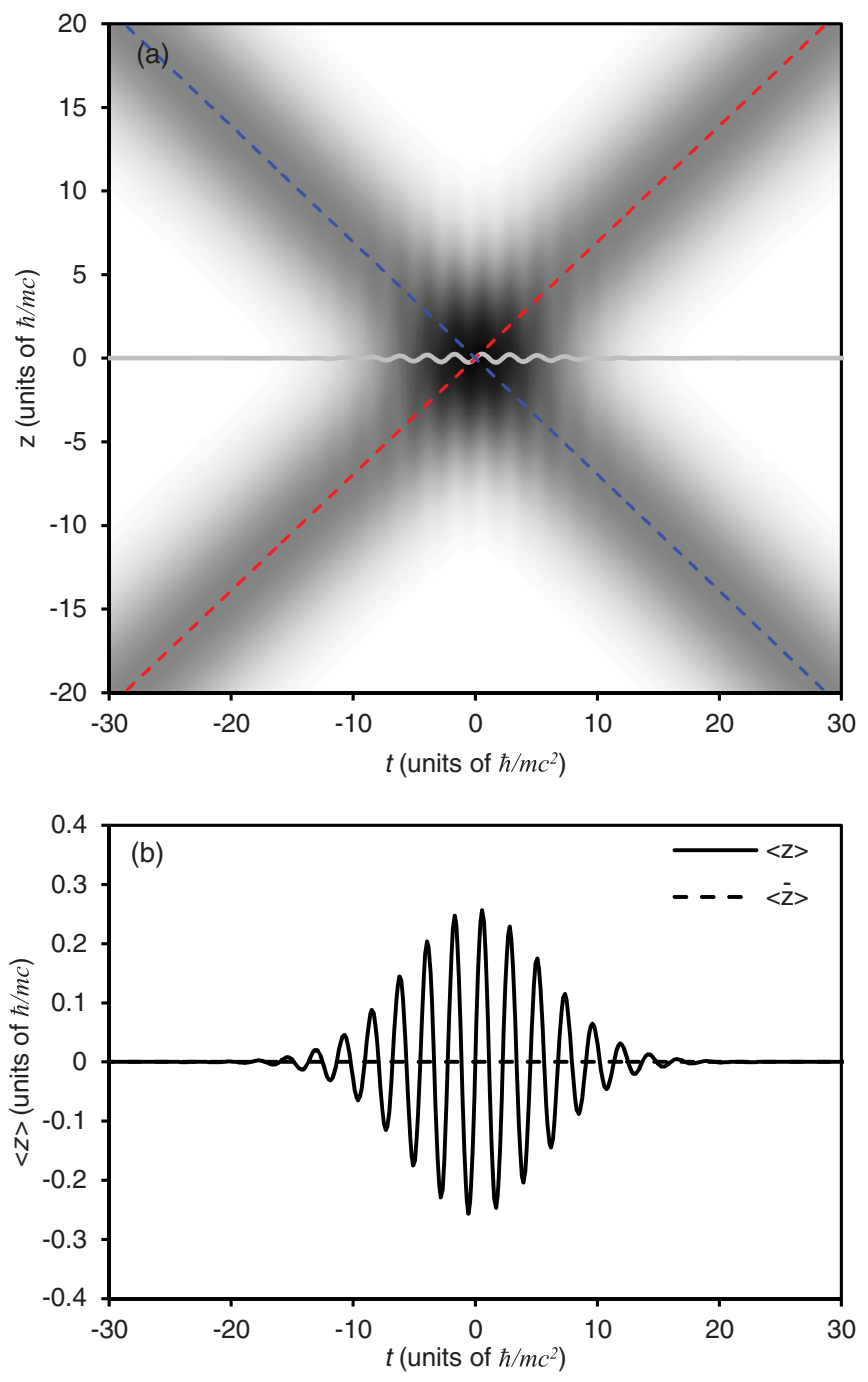

FIG. 2. (Color online) (a) Density plot of the probability and (b) line plot of the position expectation value of a total $(1+1)$ mixed wave function $(\Theta=\pi / 4)$ with $p_{0}=0.967 \mathrm{mc}$ and $2 \sigma_{p}=0.25 \mathrm{mc}$. Red (light gray) dashed, blue (dark gray dashed), and gray solid lines indicate the position expectation values of positive-energycomponent-only, negative-energy-component-only, and total mixed wave packets, respectively.

The $\sigma_{z}^{-2}$ term in Eq. (51) is due to the normalization correction term, which results from truncation of higher-order Taylor expansions. It reflects attenuation of $f^{+}$and $f^{-}$from Eqs. (40) and (41) when all the terms are included in Eq. (38) [see Eq. (A1)]. Here, we note that Eq. (50) is not a single-frequency oscillation, especially for significant momentum spreads, and $\omega_{Z B} \approx 2 \omega_{0}$ from the linear term in Eq. (50) is only an approximation (see Sec. S.I C in the Supplemental Material). For $t \rightarrow 0$ and $p_{0} \rightarrow 0$, we can approximate as $d \ll \sigma_{z}$ and $\phi \approx \frac{d}{\sigma_{z}}$, and the angular frequency of the oscillation becomes

$$
\omega_{Z B} \rightarrow\left\{1+\frac{1}{2 \sigma_{z}^{2}}\left(\frac{\lambda_{C}}{2 \gamma_{0}^{2}}\right)^{2}\right\} 2 \omega_{0} .
$$

For $t \rightarrow \infty$, we can approximate as $\Sigma_{z} \approx d$ and $\phi=\pi / 4$, and the angular frequency of the oscillation becomes

$$
\omega_{Z B} \rightarrow\left(3-\gamma_{0}^{2}\right) \omega_{0} .
$$

Note that the temporal decay is governed by the overlap between two dispersing wave packets as $\sqrt{\frac{\sigma_{z}}{\Sigma_{z}}} \exp \left[-\frac{v_{0}^{2} t^{2}}{2 \Sigma_{z}^{2}}\right]$, and the Zitterbewegung behavior vanishes due to the temporal overlap term at $t \rightarrow \infty$ for $\gamma_{0}>1$. Using Eqs. (51) and (52), the maximum speed becomes

$$
v_{Z B}=A_{Z B} \omega_{Z B} \rightarrow \frac{c}{\gamma_{0}} \frac{1+\frac{\lambda_{C}^{2}}{8 \sigma_{z}^{2} \gamma_{0}^{4}}}{1+\frac{\hbar_{C}^{2}}{16 \sigma_{z}^{2} \gamma_{0}^{4}}}
$$

at $t=0$. Equation (54) becomes $\frac{c}{\gamma_{0}}$ for $\sigma_{z} \gg \lambda_{C}$. Even though Eq. (54) reflects well the relativistic effect of the Zitterbewegung behavior ( $p_{0}$ dependence), it also predicts $v_{Z B}>c$ for $p_{0}=0$, which is relativistically impossible. We infer that it is an artifact due to a first-order approximation invoked to derive our analytical solution.

For a given mass (and $2 \sigma_{p} \ll m c$ ), in the far relativistic limit of $\gamma_{0} \rightarrow \infty$, the amplitude and the speed vanish, whereas in the nonrelativistic limit of $\gamma_{0} \rightarrow 1$, the amplitude becomes $\lambda_{C} / 2$, the angular frequency becomes $2 c / \lambda_{C}$, and the speed becomes $c$. For a given (nonzero) momentum, on the other hand, in the relativistic limit of $m \rightarrow 0$ and $\lambda_{C} \rightarrow \infty$, it becomes $\gamma_{0} \approx$ $\frac{p_{0}}{m c} \rightarrow \infty, A_{Z B} \rightarrow \frac{\hbar m c}{2 p_{0}^{2}} \rightarrow 0$, and $\omega_{Z B} \rightarrow 2 \frac{p_{0} c}{\hbar}$ (which agrees with $\hbar \omega=p c$ for a massless particle) and $v_{Z B} \rightarrow \frac{m c^{2}}{p_{0}} \rightarrow 0$, whereas in the nonrelativistic limit of $m \rightarrow \infty$ and $\lambda_{C} \rightarrow 0$, it becomes $\gamma_{0}=1$ and $A_{Z B}=\frac{\hbar}{2 m c} \rightarrow 0$ but $v_{Z B} \rightarrow c$.

\section{B. Validation of Zitterbewegung behavior: Numerical simulations}

In the previous section, we derived an analytical expression of Zitterbewegung behavior in Dirac-like systems, using our analytical solutions of Dirac wave packets. Here, in order to verify it, we compare it to (exact) numerical simulations. It is noteworthy that the analytical expression provides a physical insight which is lost in numerical simulations, although the Dirac equation can be solved exactly in numerical simulation, whereas our analytical solutions are a first-order approximation.

In many studies of Zitterbewegung behavior, an equivalent to the Dirac equation, Eq. (11), was directly solved numerically. The Dirac equation for free wave packets can be numerically solved using Eq. (11), or (36), with the initial condition given by Eq. (32) or (34). The benefit of using the differential equations for envelope functions, Eqs. (36) and (37), over others is that the carrier wave $e^{i\left(k_{0} z-\omega_{0}^{ \pm} t\right)}$ is factored out, and therefore a differential equation is solved for slowly varying parts only. Note that $\omega_{0}^{ \pm}$is large even for $k_{0}=0$. For free particles, alternatively, the wave function can be directly evaluated. When the wave function in momentum space at $t=0$ is given by

$$
\Psi(k, 0)=\hat{\boldsymbol{u}}_{(k)}^{+} \hat{f}^{+}(k)+\hat{\boldsymbol{u}}_{(k)}^{-} \hat{f}^{-}(k),
$$

the wave function at any time becomes

$$
\Psi(k, t)=\hat{\boldsymbol{u}}_{(k)}^{+} \hat{f}^{+}(k) \boldsymbol{e}^{-i \omega^{+}(k) t}+\hat{\boldsymbol{u}}_{(k)}^{-} \hat{f}^{-}(k) \boldsymbol{e}^{-i \omega^{-}(k) t},
$$

and the wave function in position space at $t$ is obtained by inverse Fourier transformation as $\Psi(z, t)=\mathcal{F}^{-1}\{\Psi(k, t)\}$. 

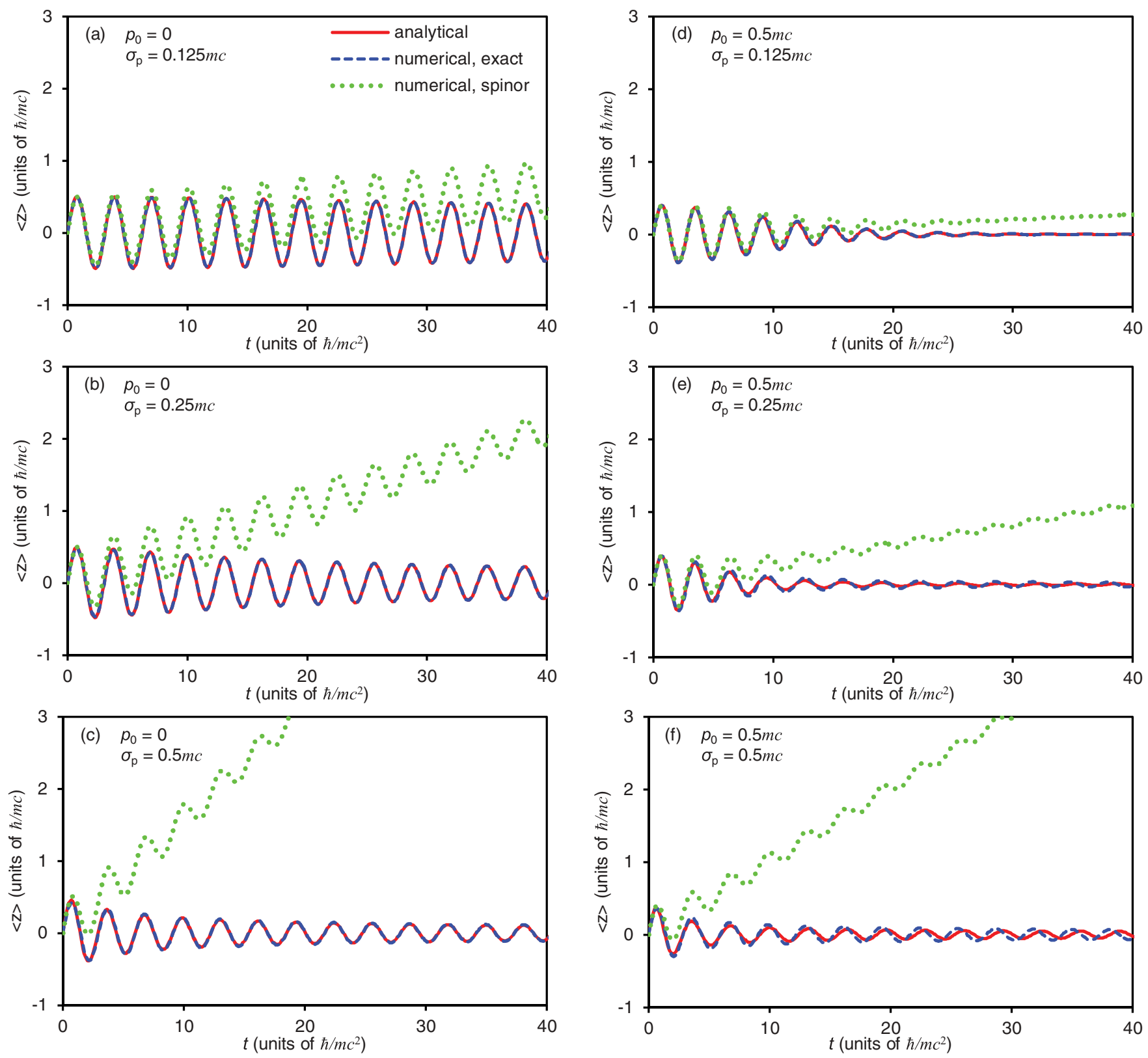

FIG. 3. (Color online) Comparison of the position expectation values from the analytical solution (red solid line) and the numerical simulations of the exact mixed state (blue dashed line) and the spinor state (green dotted line) at $\Theta=\pi / 4$, with $p_{0}$ and $\sigma_{p}$ of (a) $0,0.125$, (b) $0,0.25$, (c) $0,0.5$, (d) $0.5,0.125$, (e) $0.5,0.25$, and (f) $0.5,0.5$ in units of $m c$. Note that spinor packets show a drift due to momentum shift.

Figures 3(a)-3(c) compare the analytical expression, Eq. (50), and the numerical simulation with the exact Gaussian packets, Eq. (24), and its negative-energy counterpart with $p_{0}=0$ and equal populations $\left(\Theta=\frac{\pi}{4}\right)$. Because the positiveand the negative-energy wave packets are at rest $\left(v^{+}=v^{-}=\right.$ $0)$, the position expectation value for the total wave function always shows an interference pattern, whose magnitude attenuates as wave packets disperse [the $\sqrt{\frac{\sigma_{z}}{\Sigma_{z}}}$ term in Eq. (50)]. The analytical expression and the exact numerical simulation show an excellent agreement. The agreement between the first-order analytical solution and the exact numerical simulation proves that our analytical solution, even though it is a first-order approximation, is correct. Also compared is the numerical simulation with the spinor wave packets as the initial state, which exhibits a drift (see Sec. IV B for a discussion on spinor states). The drift behavior is due to the fact that the spinor wave packets mixes positive- and negative-energy components, and a $(1,1)$ spinor packet generates the positiveand the negative-energy components blue- and redshifted, respectively, in momentum space, which becomes more severe as the momentum distribution becomes larger.

Figures 3(d)-3(f) compare cases for $p_{0}=0.5 m c$ with equal populations of the positive- and the negative-energy components $\left(\Theta=\frac{\pi}{4}\right)$. The position expectation value for the total wave function shows an interference pattern when they overlap and vanishes when they propagate apart [the $\exp \left[-\frac{v_{0}^{2} t^{2}}{2 \Sigma_{z}^{2}}\right]$ term in Eq. (50)]. Even though their agreement is fairly good, the analytical expression, which is only a 
first-order solution, deviates from the exact simulation for the cases of large momentum spreads due to neglecting higherorder terms. When $\sigma_{p}$ is large, dispersion becomes greater than propagation, and the interference does not vanish. In particular, the linear approximation employed here [Eq. (38)] implies that the center-of-momentum distribution corresponds to the center-of-velocity distribution, and the propagation is determined only by the second terms in Eqs. (36) and (37), and the third terms therein only contribute to dispersion. However, when $2 \sigma_{p} \gg m c$ and $\sigma_{z} \ll \lambda_{C}$, higher-order terms need to be explicitly considered in Eq. (38), which results in a modified propagation. It should be noted that the velocity cannot be larger than $c$ in relativity.

Gerritsma et al. [13] performed the experiment of position measurement of trapped ions in a Dirac-like environment and compared it to the numerical simulation of wave-packet propagation. Since the positions of ions in the upper and lower state are no longer subject to the dilemma that a particle cannot be in both matter and antimatter states (see Sec. IV C), their observation of a trembling motion of the trapped ion is valid. In Fig. 2 of Ref. [13], the speed of light and the reduced Compton wavelength are given as $c=0.52 \Delta / \mu \mathrm{s}$ and $\lambda_{C}=0.62 \Delta$, respectively. Therefore, it corresponds to $\sigma_{z}=\Delta=1.6 \lambda_{C}$ and $k_{0}=\frac{1}{\Delta}=0.62 \lambda_{C}^{-1}$, which corresponds to $\gamma_{0}=1.177$. Then the $(1,1)$ spinor corresponds to 0.76 and 0.24 populations of positive- and negative-energy components $(\Theta=0.5076)$. Since they are not of equal populations, the position expectation value also propagates over time by the $v_{0} t\left(\cos ^{2} \Theta-\sin ^{2} \Theta\right)$ term in Eq. (50), as plotted in Fig. 4. Also plotted are their original data and simulation, which were converted to values in $\lambda_{C}$ and $\lambda_{C} / c$, using their definitions of $\lambda_{C}$ and $c$. They show a generally good agreement. In Eq. (50), the angular frequency of Zitterbewegung behavior is approximately given by $\omega_{Z B} \approx 2 \omega_{0}=2 \gamma_{0} \frac{m c^{2}}{\hbar}$. In Fig. 1 of Ref. [13], the authors examined a mass dependence of Zitterbewegung frequency and amplitude. In particular, they deduce that $\omega_{Z B} \approx 2 \Omega$ from numerical simulations. In

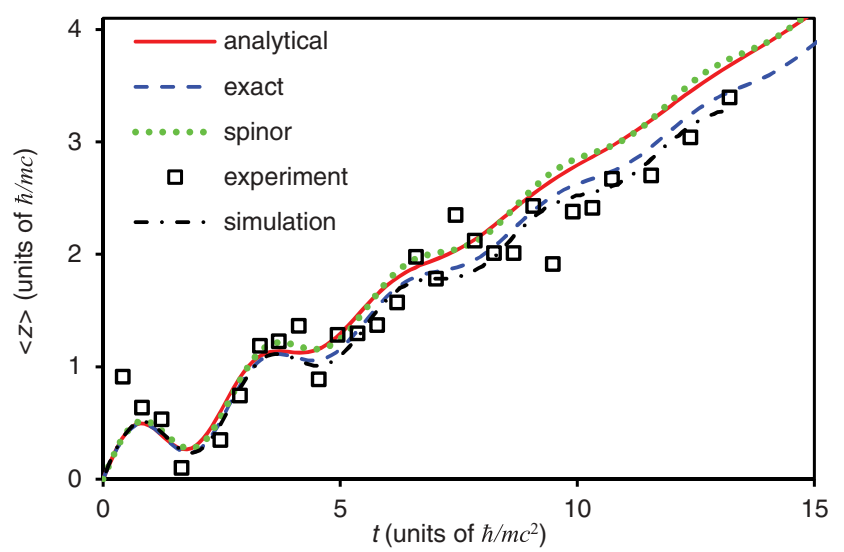

FIG. 4. (Color online) Position expectation values of the total mixed wave function $(\Theta=0.5076)$ from the analytical solution (red solid line) and the numerical simulations of the exact mixed state (blue dashed line) and the (1,1) spinor state (green dotted line), where $\sigma_{z}=\Delta=\frac{\lambda_{C}}{0.62}$ and $k_{0}=\frac{1}{\Delta}$. Also plotted are the experimental (black squares) and simulated (black center line) mean positions in Ref. [13] (digitized from Fig. 2 therein). their work, it is given that $m c^{2}=\hbar \Omega$ and $\lambda_{C}=2 \eta \tilde{\Omega} \Delta / \Omega$. Therefore, Eq. (53) predicts that the angular frequency of oscillation becomes $\omega_{Z B}=2 \Omega$ for $p_{0}=0$ and longer periods, which agrees with their deduction.

\section{DISCUSSION}

\section{A. Closer look at Zitterbewegung behavior: Limitation of analytical solutions}

As discussed in the previous section, Eqs. (51) and (52) are only expected to hold true for $2 \sigma_{p} \ll m c$. Figure 5(a) compares Eq. (52) with the angular frequency obtained by fitting the numerical simulations with exact Gaussian packets
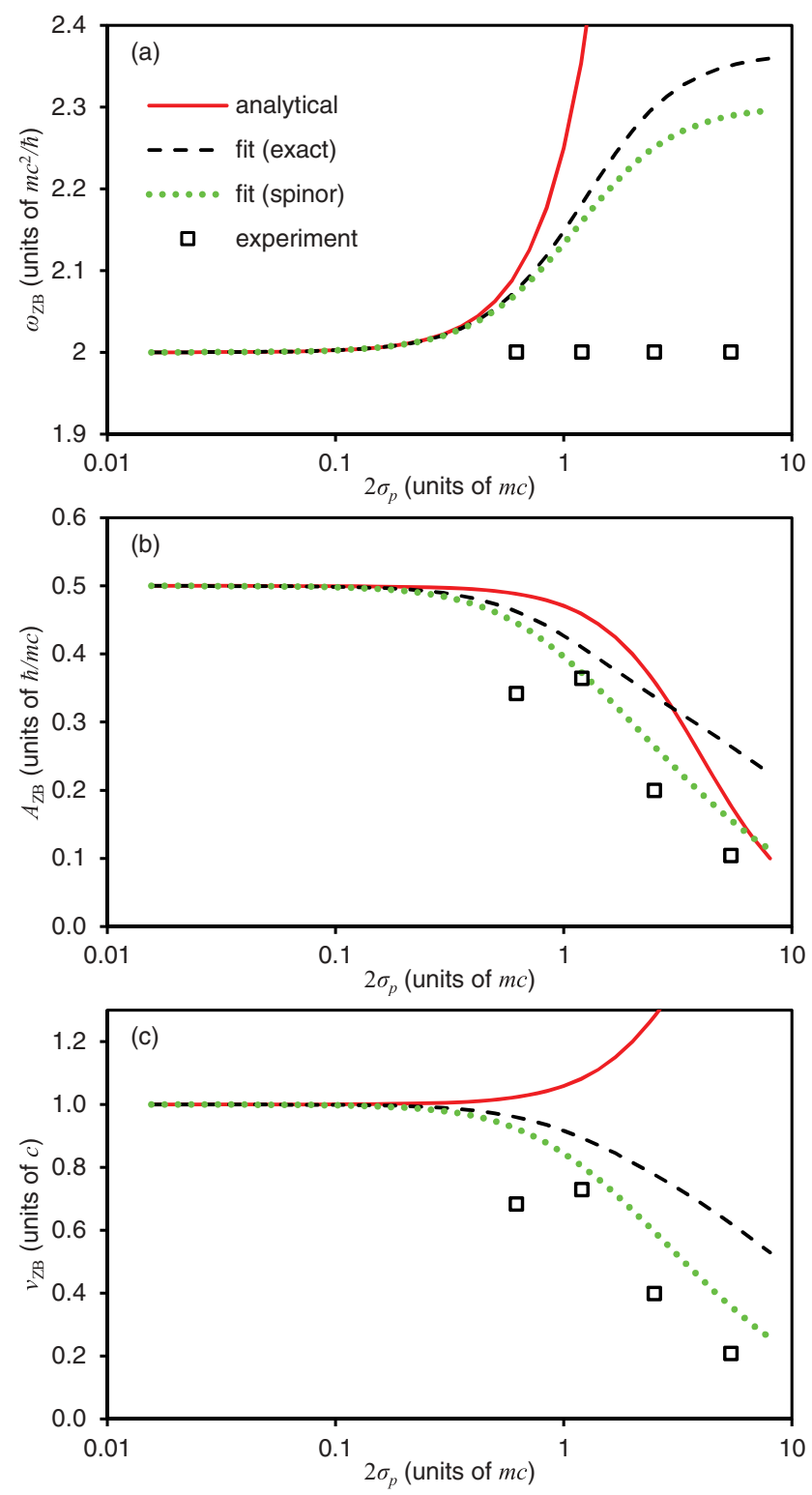

FIG. 5. (Color online) (a) The initial angular frequencies, (b) the amplitudes, and (c) the maximum velocities of the Zitterbewegung behavior of the analytical solution (red solid line), the numerical simulations with exact Gaussian packets (black dashed line), and spinor wave packets (green dotted line) as a function of momentum spread at $p_{0}=0$. Also plotted are the experimental values (black squares) from Ref. [13] (digitized from their Fig. 1 inset). 
$\left(p_{0}=0\right)$ with a simple attenuated sinusoidal function for the first period of oscillation $\left(|t| \leqslant \pi \omega_{0}^{-1}\right)$. Equation (52) increases from $2 \omega_{0}$ for larger momentum spreads due to the $\phi$ term, and it agrees with the numerical simulation for $2 \sigma_{p}<0.5 m c$. We note that Eq. (50) is not a single-frequency oscillation, and when we evaluate the angular frequency for $t \gg 2 \pi \omega_{0}^{-1}$, we obtain $\omega_{Z B}=2.00 \omega_{0}$, as predicted from Eq. (53) for $p_{0}=0$. Figure 5(b) compares Eq. (51) with the amplitude obtained by fitting the numerical simulations. Since the oscillation attenuates as wave packets disperse, it is not straightforward to extract a true amplitude without knowledge of the attenuation. We used the dispersive attenuation from Eq. (50) with $\sigma_{v}$ determined by fitting. Equation (51) decreases as the momentum spread increases, and its trend agrees with numerical simulations. Figure 5(c) compares Eq. (54) with the maximum velocity obtained by fitting the numerical simulations. Equation (54) predicts $v_{Z B}>c$ for $p_{0}=0$, which is unphysical (see Sec. III A), whereas that from fitting numerical simulations is less than $c$ and further decreases for larger momentum spreads. We attribute this to the observation that the analytical amplitude by Eq. (51) is similarly overestimated in Fig. 5(b). A further examination reveals that it is because the dispersion, $\sigma_{v}=\frac{\sigma_{p}}{\gamma_{0}^{3} m}$, is only valid for a very small $\sigma_{p}$ (see Figure S.2 in the Supplemental Material).

The frequencies, amplitudes, and velocities fitted from spinor wave packet simulation slightly deviate from those for exact Gaussian packet simulations, which we attribute to the momentum shift in spinor wave packets (see Sec. IV B). Gerritsma et al. observed that the amplitude of the Zitterbewegung behavior decreases as the effective mass decreases and explained it as a far-relativistic behavior. However, we note that by making $m \rightarrow 0$, we get $\lambda_{C}=\frac{\hbar}{m c} \rightarrow \infty$, and therefore $A_{Z B} \rightarrow \infty$ as long as $\sigma_{z} \gg \lambda_{C}$. On the other hand, Eq. (51) predicts that $A_{Z B} \rightarrow 0$ when $\sigma_{z} \ll \lambda_{C} \rightarrow \infty$ or $2 \sigma_{p} \gg m c \rightarrow 0$, which is the case for a fixed $\sigma_{z}$. Therefore, we infer that it is a momentum spread effect. However, we also recognize that with a large momentum spread it has significant populations for $|p|>m c$, which is in a relativistic regime.

\section{B. Spinor wave packet}

The so-called spinor wave packet is often employed for an initial state in numerical simulations, where a wave packet is generated by multiplying a wave-packet profile in position space $\sqrt{G(z)}$ and a single-energy planar wave function $\hat{\boldsymbol{u}}_{\left(k_{0}\right)}^{ \pm} e^{i k_{0} z}$. However, this, in fact, generates a state with mixed positive- and negative-energy components because it neglects the $k$ dependence of spinor coefficients in Eq. (24) (also see Sec. S.I A in the Supplemental Material). Thaller [35] acknowledges that a pure Gaussian packet in position space mixes positive- and negative-energy states (see Fig. 7.4 in Ref. [35]). However, in the study of the Zitterbewegung, a wave packet is prepared in that manner, which is only a zeroth-order approximation. We note that Zitterbewegung behavior in the position expectation value of the total wave function arises from the fact that Dirac wave packets consist of major components $\left(f_{e}^{+}\right.$and $\left.f_{p}^{-}\right)$and minor components $\left(f_{e}^{-}\right.$ and $f_{p}^{+}$), not because the zeroth-order solution mixes positiveand negative-energy components.
However, the latter is attributed to the following: Thaller [36] observed a peculiar behavior besides Zitterbewegung, namely, that mixed-state wave packets prepared with zero momenta slowly drift in a positive direction. We note that this is an artifact due to a zeroth-order solution (spinor wave packet). When the initial state is prepared, like one would do in Schrödinger equations, as

$$
\begin{aligned}
& \Phi(z, 0)=C^{\prime} \exp \left[-\frac{z^{2}}{4 \sigma_{z}^{2}}\right]\left(\begin{array}{c}
\cos \Omega \\
\sin \Omega
\end{array}\right) e^{i k_{0} z}, \\
& \Phi(k, 0)=C \exp \left[-\frac{\left(k-k_{0}\right)^{2}}{4 \sigma_{k}^{2}}\right]\left(\begin{array}{c}
\cos \Omega \\
\sin \Omega
\end{array}\right),
\end{aligned}
$$

where $\Omega$ is a given constant value, it corresponds to shifted momentum distributions as follows: the constant spinor in Eq. (58) can be approximated as

$$
\begin{aligned}
\left(\begin{array}{c}
\cos \Omega \\
\sin \Omega
\end{array}\right)= & \hat{\boldsymbol{u}}_{\left(k_{0}\right)}^{+} \cos \Theta+\hat{\boldsymbol{u}}_{\left(k_{0}\right)}^{-} \sin \Theta \\
\approx & \left\{\hat{\boldsymbol{u}}_{(k)}^{+}-\left(k-k_{0}\right) \frac{\lambda_{C}}{2 \gamma_{0}^{2}} \hat{\boldsymbol{u}}_{\left(k_{0}\right)}^{-}\right\} \cos \Theta \\
& +\left\{\hat{\boldsymbol{u}}_{(k)}^{-}+\left(k-k_{0}\right) \frac{\lambda_{C}}{2 \gamma_{0}^{2}} \hat{\boldsymbol{u}}_{\left(k_{0}\right)}^{+}\right\} \sin \Theta,
\end{aligned}
$$

and the spinor wave packet $\Phi$ can be decomposed into pure energy components (see Appendix D) as

$$
\begin{aligned}
\hat{f}^{+}(k)= & \left(\hat{\boldsymbol{u}}_{(k)}^{+}\right)^{T} \Phi(k) \\
= & C \exp \left[-\frac{\left(k-k_{0}-\gamma_{0}^{-2} \lambda_{C} \sigma_{k}^{2} \tan \Theta\right)^{2}}{4 \sigma_{k}^{2}}\right] \\
& \times \exp \left[-\left(\frac{\lambda_{C}}{2 \gamma_{0}^{2}} \sigma_{k} \tan \Theta\right)^{2}\right] \cos \Theta, \\
\hat{f}^{-}(k)= & \left(\hat{\boldsymbol{u}}_{(k)}^{-}\right)^{T} \Phi(k) \\
= & C \exp \left[-\frac{\left(k-k_{0}+\gamma_{0}^{-2} \lambda_{C} \sigma_{k}^{2} \cot \Theta\right)^{2}}{4 \sigma_{k}^{2}}\right] \\
& \times \exp \left[-\left(\frac{\lambda_{C}}{2 \gamma_{0}^{2}} \sigma_{k} \cot \Theta\right)^{2}\right] \sin \Theta .
\end{aligned}
$$

It is observed that $\hat{f}^{+}(k)$ is shifted by $+\gamma_{0}^{-2} \lambda_{C} \sigma_{k}^{2} \tan \Theta$ and $\hat{f}^{-}(k)$ is shifted by $-\gamma_{0}^{-2} \lambda_{C} \sigma_{k}^{2} \cot \Theta$. It is to be noted that the momentum shifts are quadratically proportional to the momentum spread. Since the velocities of an electron and a positron are given by $+\frac{p}{\gamma m}$ and $-\frac{p}{\gamma m}$, respectively, and their effective momentum shifts are $\delta p^{ \pm}= \pm(\tan \Theta)^{ \pm 1} \gamma_{0}^{-2} \hbar \lambda_{C} \sigma_{k}^{2}=$ $\pm(\tan \Theta)^{ \pm 1} \sigma_{p}^{2} / \gamma_{0}^{2} m c$, they both drift in the same direction with a velocity of $\delta v^{ \pm}=(\tan \Theta)^{ \pm 1} \sigma_{p}^{2} / \gamma_{0}^{3} m^{2} c$, respectively.

$f^{+}(k)$ and $f^{-}(k)$ are plotted for $(1,0)$ and $(1,1)$ spinor wave packets with $k_{0}=0$ in Fig. 6 . When the $(1,0)$ spinor is used, it results in contamination by a small negativeenergy component, and the distribution of the positive-energy component is centered at $p=0$. On the other hand, when the $(1,1)$ spinor is used, the positive-energy component is blueshifted, and the negative-energy component is redshifted, resulting in both components drifting with a positive velocity. Figures 3(a)-3(c) show this drift for the $(1,1)$ spinor wave 

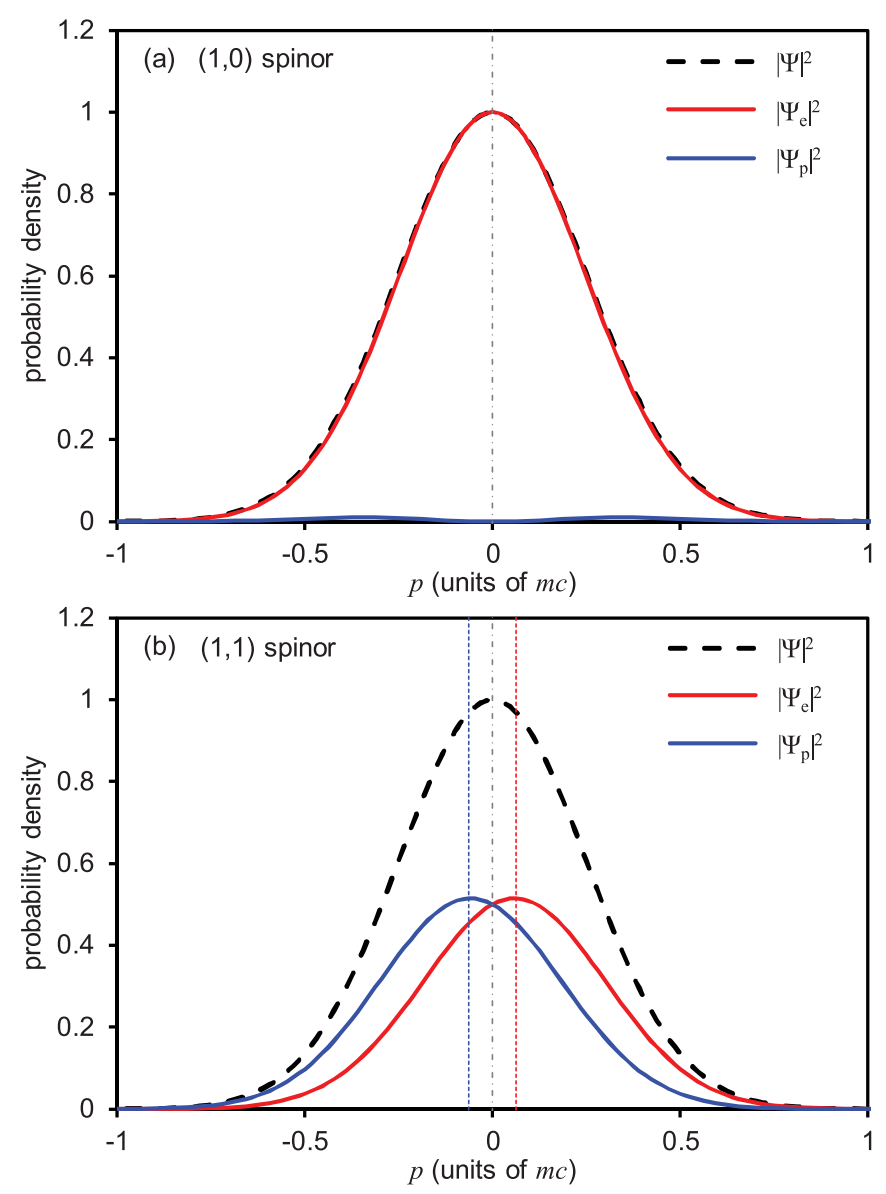

FIG. 6. (Color online) Momentum profiles of total (black dashed line), positive-energy [red (light gray) solid line], and negative-energy [blue (dark gray) solid line] components of spinor-basis wave packets prepared with $\sigma_{p}=0.25 m c$ in (a) $(1,0)$ and (b) $(1,1)$ spinors. Vertical lines indicate momentum shifts, calculated using Eqs. (60) and (61).

packet for $p=0$ and $\sigma_{p}=0.125 m c, 0.25 m c$, and $0.5 m c$. Note that the drift is a result of the momentum shifts in the spinor wave packets, and the exact solutions do not exhibit it.

\section{Dirac equation vs Dirac-like system}

The question on the physical nature of the superposition state of the positive- and negative-energy solutions in the Dirac equation, which is necessary for the Zitterbewegung to exist, is rather philosophical and beyond the scope of this paper. Here, our main objective is to derive an analytical solution, when such a superposition is allowed, which provides a physical insight on the mechanism of Zitterbewegung. Furthermore, our result is directly applicable to the observations in Dirac-like systems since they are not subject to the same question. The fact that the positive- and negative-energy-only wave functions and their superposition describe well both the expected behavior of individual wave packets and the unexpected behavior of Zitterbewegung suggests that our method and solution thus obtained are valid.

Nevertheless, it is pertinent to mention a difference between the Dirac equation and the Dirac-like system. Both are single-particle equations, but the positive- and negative-energy components correspond to the upper and lower states of one particle, a trapped ion, in the Dirac-like system, whereas they correspond to the states of two different particles, the electron and the positron, in the Dirac equation. That is, even though the Dirac equation is a single-particle formalism, it applies to and describes two different types of particles, electrons and positrons, simultaneously. We conjecture that unless an electron is allowed to occupy the negative-energy state, which will follow the opposite equation of motion, the superposition seems unphysical. We argue that a positiveor negative-energy-only wave function does not violate the completeness of wave-function space because they belong to two different types of particles [31,32]. In our opinion, a matter particle is allowed to occupy only the positive-energy states, and an antimatter particle is allowed to occupy only the negative-energy states, perhaps similar to a total wave function of bosons only being allowed to be symmetric with respect to permutation and one for fermions to be antisymmetric. We also add that in a previous work [30], it was (approximately) shown that the positive-energy wave function is sufficient to describe the relativistic electron after interaction with a light. In quantum field theory, a superposition of positiveand negative-energy wave functions is deemed physically impossible. However, a hole theory [6,37] describes that a hole can be seen as a positron, and Wang and Xiong [11] argued that therefore quantum field theory may still allow the existence of Zitterbewegung.

In a Dirac-like system [13-18], a superposition of the lower and the upper states of a single particle raises no issues, and therefore Zitterbewegung behavior is valid. However, its relation to the proof of the Zitterbewegung in the Dirac equation is subject to whether it is possible to superpose positive- and negative-energy solutions to describe an electron wave function or not.

\section{SUMMARY}

The Dirac equation for a free electron was theoretically studied using an envelope-function method. To first order for the small momentum spread, the correspondence to nonrelativistic wave-packet propagation was derived and was found to be a relativistic correction of $\gamma_{0}^{-3}$ to dispersion. Furthermore, it was demonstrated that using a constant spinor is not sufficient, and it is crucial to include at least the firstorder term to correctly describe Dirac packets. An analytical expression for either electron or positron wave packets was obtained separately. It was demonstrated that a pure-energy single-particle wave packet in the Dirac equation does not exhibit any trembling motion. Using those analytical solutions, the Zitterbewegung behavior of a hypothetical superposition state was evaluated, and we established that it is the first-order term that dictates the Zitterbewegung behavior.

Our results using the analytical wave packets show excellent agreement with the Zitterbewegung behavior in numerical simulations, in particular, when the momentum spread is not too great. When numerical simulations are invoked, the Zitterbewegung behavior is analyzed by fitting the numerical results with a sinusoidal function. However, it was demonstrated that the angular frequency obtained thus depends on how much of the numerical results are used for fitting because the Zitterbewegung behavior is not a single-frequency oscillation, as often 
assumed. Also, with numerical simulations, spinor wave packets, which are only a zeroth approximation, are often employed as initial states. Apparent outcomes of using spinor wave packets can also be estimated from our result and were discussed in terms of population mixing, momentum shift, and drift.

\section{ACKNOWLEDGMENTS}

This work was supported by the National Science Foundation and the Air Force Office of Scientific Research in the Center for Physical Biology funded by the Gordon and Betty Moore Foundation. The author is grateful to Professor A. H. Zewail for his support. The author also would like to thank Jessica A. Pfeilsticker for the helpful discussion.

\section{APPENDIX A: HIGH-FREQUENCY APPROXIMATION}

First, we designate $\omega_{0} \equiv+\omega_{0}^{+}=-\omega_{0}^{-}$, such that $\omega_{0}^{+}-$ $\omega_{0}^{-}=2 \omega_{0}$. An approximate solution for $f^{-}(z, t)$ in Eq. (37) can be obtained by assuming that the temporal behavior of $f^{-}(z, t)$ is dominated by the fast oscillation terms, $\exp \left[-2 i \omega_{0} t\right]$, and any cumulated value is quickly dissipated due to the $-v_{0}$ propagation term; therefore the only surviving term is from $f^{+}(z, t) \exp \left[-2 i \omega_{0} t\right]$. In other words, any change in $f^{-}(z, t)$ is propagated in the opposite direction from $f^{+}(z, t)$, and therefore the solution of $f^{-}(z, t)$ that propagates along with $f^{+}(z, t)$ is very small and can be approximated as

$$
\begin{aligned}
f^{-}(z, t)= & -\sum_{n=0}^{\infty}\left(\frac{1}{-2 i \omega_{0}}\right)^{n+1} \frac{c}{\gamma_{0}}\left\{\left(-\frac{\partial}{\partial t}\right)^{n} \frac{\partial f^{+}}{\partial z}\right\} \\
& \times \exp \left[-2 i \omega_{0} t\right] \\
= & -\frac{1}{-2 i \omega_{0}} \frac{c}{\gamma_{0}}\left(\frac{\partial f^{+}}{\partial z}\right) \exp \left[-2 i \omega_{0} t\right]+O\left(\omega_{0}^{-2}\right) \\
\approx & -\frac{1}{-2 i \omega_{0}} \frac{c}{\gamma_{0}}\left(\frac{\partial f^{+}}{\partial z}\right) \exp \left[-2 i \omega_{0} t\right],
\end{aligned}
$$

which approximately satisfies the major component part,

$$
\frac{\partial f^{-}}{\partial t}+\frac{c}{\gamma_{0}}\left(\frac{\partial f^{+}}{\partial z}\right) e^{-2 i \omega_{0} t} \approx 0,
$$

for very large $\omega_{0}$ and relatively slowly varying $f^{+}(z, t)$ (highfrequency approximation).

\section{APPENDIX B: MEAN POSITION OF TOTAL MIXED STATE}

The position expectation value of Eq. (48) is given by

$$
\begin{aligned}
\langle z\rangle & \equiv\left\langle\Psi^{\dagger}|z| \Psi\right\rangle \\
& =\frac{v_{0} t\left(1+\frac{\lambda_{C}^{2}}{16 \sigma_{z}^{2} \gamma_{0}^{4}}\right)\left(\cos ^{2} \Theta-\sin ^{2} \Theta\right)+\frac{\lambda_{C}}{2 \gamma_{0}^{2}} \sqrt{\frac{\sigma_{z}}{\Sigma_{z}}} \exp \left[-\frac{v_{0}^{2} t^{2}}{2 \Sigma_{z}^{2}}\right] \sin \left(2 \omega_{0} t-\frac{\lambda_{C} v_{0}^{2} c t^{3}}{4 \gamma_{0}^{3} \sigma_{z}^{2} \Sigma_{z}^{2}}+\frac{1}{2} \phi\right) 2 \sin \Theta \cos \Theta}{1+\frac{\lambda_{C}^{2}}{16 \sigma_{z}^{2} \gamma_{0}^{4}}} \\
& =v_{0} t \cos 2 \Theta+\left\{1+\frac{\lambda_{C}^{2}}{16 \sigma_{z}^{2} \gamma_{0}^{4}}\right\}^{-1} \frac{\lambda_{C}}{2 \gamma_{0}^{2}} \sqrt{\frac{\sigma_{z}}{\Sigma_{z}}} \exp \left[-\frac{v_{0}^{2} t^{2}}{2 \Sigma_{z}^{2}}\right] \sin \left(2 \omega_{0} t-\frac{\lambda_{C} v_{0}^{2} c t^{3}}{4 \gamma_{0}^{3} \sigma_{z}^{2} \Sigma_{z}^{2}}+\frac{1}{2} \phi\right) \sin 2 \Theta \\
& =v_{0} t \cos 2 \Theta+\left\{1+\frac{\lambda_{C}^{2}}{16 \sigma_{z}^{2} \gamma_{0}^{4}}\right\}^{-1} \frac{\lambda_{C}}{2 \gamma_{0}^{2}} \sqrt{\frac{\sigma_{z}}{\Sigma_{z}}} \exp \left[-\frac{v_{0}^{2} t^{2}}{2 \Sigma_{z}^{2}}\right] \sin \left[2 \omega_{0} t-\frac{d^{2}}{\Sigma_{z}^{2}}\left(\gamma_{0}^{2}-1\right) \omega_{0} t+\frac{\phi}{2}\right] \sin 2 \Theta,
\end{aligned}
$$

where $2 \omega_{0}=\omega_{0}^{+}-\omega_{0}^{-}, \Sigma_{z}=\sqrt{\sigma_{z}^{2}+d^{2}}, \phi=\tan ^{-1}\left(\frac{d}{\sigma_{z}}\right)$, and $d=\sigma_{v} t=\frac{\hbar t}{2 \gamma_{0}^{3} m \sigma_{z}}$. We also utilized the relations $\frac{v_{0}}{c}=\sqrt{1-\gamma_{0}^{-2}}$ and $\omega_{0}=\gamma_{0} \frac{m c^{2}}{\hbar}$.

\section{APPENDIX C: SPINOR PROJECTION}

The projection operator (in momentum space) is defined as

$$
\hat{P}_{ \pm}=\frac{1}{2}\left(1 \pm \frac{c \alpha \cdot \hat{p}+\beta m c^{2}}{\sqrt{\hat{p}^{2} c^{2}+m^{2} c^{4}}}\right) .
$$

However, an implicit single-energy spinor projection in position space is often invoked in analyzing numerical simulation results, due to its simplicity, to separate positive- and negative-energy components and generate a so-called spinor state. The single-energy spinor projections onto the positive- and the negative-energy components become

$$
\begin{aligned}
\Phi_{+}(z, t) & =\hat{\boldsymbol{u}}_{\left(k_{0}\right)}^{+}\left(\hat{\boldsymbol{u}}_{\left(k_{0}\right)}^{+}\right)^{T} \cdot \Psi(z, t) \\
& =\hat{\boldsymbol{u}}_{\left(k_{0}\right)}^{+}\left\{f_{e}^{+}(z, t) \exp \left[i\left(k_{0} z-\omega_{0}^{+} t\right)\right] \cos \Theta+\frac{i \hbar}{2 \gamma_{0}^{2} m c} \frac{\partial f_{p}^{-}(z, t)}{\partial z} \exp \left[i\left(k_{0} z-\omega_{0}^{-} t\right)\right] \sin \Theta\right\} \\
& =\hat{\boldsymbol{u}}_{\left(k_{0}\right)}^{+}\left\{f_{e}^{+}(z, t)+i \tan \Theta \frac{\lambda_{C}}{2 \gamma_{0}^{2}} \frac{\partial f_{p}^{-}(z, t)}{\partial z} \exp \left[2 i \omega_{0} t\right]\right\} \exp \left[i\left(k_{0} z-\omega_{0}^{+} t\right)\right] \cos \Theta
\end{aligned}
$$




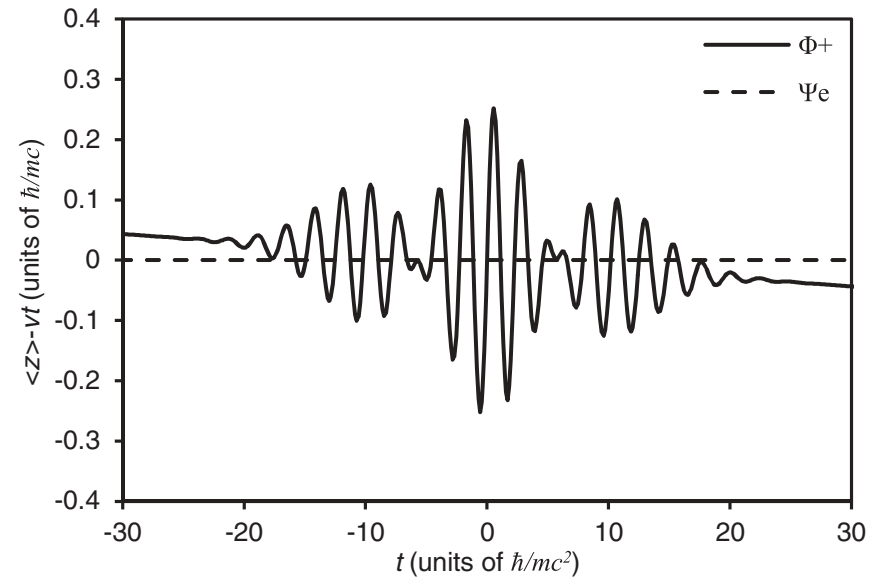

FIG. 7. Deviation of position expectation values of the spinorprojected (solid line) and the energy-projected (dashed line) wave functions with $p_{0}=0.967 m c(200 \mathrm{keV}), 2 \sigma_{p}=0.25 m c$, and $\Theta=$ $\pi / 4$. and

$$
\begin{aligned}
\Phi_{-}(z, t)= & \hat{\boldsymbol{u}}_{\left(k_{0}\right)}^{-}\left(\hat{\boldsymbol{u}}_{\left(k_{0}\right)}^{-}\right)^{T} \cdot \Psi(z, t) \\
= & \hat{\boldsymbol{u}}_{\left(k_{0}\right)}^{-}\left\{f_{p}^{-}(z, t) \exp \left[i\left(k_{0} z-\omega_{0}^{-} t\right)\right] \sin \Theta\right. \\
& \left.-\frac{i \hbar}{2 \gamma_{0}^{2} m c} \frac{\partial f_{e}^{+}(z, t)}{\partial z} \exp \left[i\left(k_{0} z-\omega_{0}^{+} t\right)\right] \cos \Theta\right\} \\
= & \hat{\boldsymbol{u}}_{\left(k_{0}\right)}^{-}\left\{f_{p}^{-}(z, t)-i \cot \Theta \frac{\lambda_{C}}{2 \gamma_{0}^{2}} \frac{\partial f_{e}^{+}(z, t)}{\partial z}\right. \\
& \left.\times \exp \left[-2 i \omega_{0} t\right]\right\} \exp \left[i\left(k_{0} z-\omega_{0}^{-} t\right)\right] \sin \Theta .
\end{aligned}
$$

It is clear that this projection mixes the contributions from the electron and the positron wave packets, $f_{e}^{+}(z, t)$ and $f_{p}^{-}(z, t)$, respectively. Then, it can be shown that the position expectation value of this projection becomes

$$
\begin{aligned}
\left\langle z_{+}\right\rangle & \equiv\left\langle\Phi_{+}^{\dagger}|z| \Phi_{+}\right\rangle \\
& =\frac{v_{0} t\left(\cos ^{2} \Theta-\frac{\lambda_{C}^{2}}{16 \sigma_{z}^{2} \gamma_{0}^{4}} \sin ^{2} \Theta\right)+\frac{\lambda_{C}}{4 \gamma_{0}^{2}} \sqrt{\frac{\sigma_{z}}{\Sigma_{z}}} \exp \left[-\frac{v_{0}^{2} t^{2}}{2 \Sigma_{z}^{2}}\right] \sin \left(2 \omega_{0} t+\frac{\lambda_{C} v_{0}^{2} c t^{3}}{4 \gamma_{0}^{3} \sigma_{z}^{2} \Sigma_{z}^{2}}+\frac{1}{2} \phi\right) \sin 2 \Theta}{\cos ^{2} \Theta+\frac{\lambda_{C}^{2}}{16 \sigma_{z}^{2} \gamma_{0}^{4}} \sin ^{2} \Theta+\frac{\lambda_{C}}{4 \gamma_{0}^{2}} \frac{v_{0} t}{\sqrt{\sigma_{z} \Sigma_{z}^{3}}} \exp \left[-\frac{v_{0}^{2} t^{2}}{2 \Sigma_{z}^{2}}\right] \sin \left(2 \omega_{0} t-\frac{\lambda_{C} v_{0}^{2} c t^{3}}{4 \gamma_{0}^{3} \sigma_{z}^{2} \Sigma_{z}^{2}}+\frac{3}{2} \phi\right) \sin 2 \Theta} .
\end{aligned}
$$

Figure 7 shows the deviation of the position expectation value of $\Phi_{+}(z, t)$ from the classical value, $\langle z\rangle=+v_{0} t$. Because the spinor-projected wave function mixes the positive- and the negative-energy components, it still shows an interference pattern. Furthermore, it deviates from the classical value, $+v_{0} t$, due to a small contamination of the negative-energy component. Therefore, it is crucial to utilize the exact projection operator, Eq. (C1), when analyzing the results of numerical simulations.

\section{APPENDIX D: SPINOR WAVE PACKET}

When the initial state is prepared as a spinor wave packet,

$$
\Phi(z, 0)=C^{\prime} \exp \left[-\frac{z^{2}}{4 \sigma_{z}^{2}}\right]\left(\begin{array}{c}
\cos \Omega \\
\sin \Omega
\end{array}\right) e^{i k_{0} z},
$$

where $\Omega$ is a given constant value, it corresponds to, in momentum space,

$$
\Phi(k, 0)=C \exp \left[-\frac{\left(k-k_{0}\right)^{2}}{4 \sigma_{k}^{2}}\right]\left(\begin{array}{c}
\cos \Omega \\
\sin \Omega
\end{array}\right) .
$$

The zeroth-order population can be evaluated by solving

$$
\left(\begin{array}{c}
\cos \Omega \\
\sin \Omega
\end{array}\right)=\hat{\boldsymbol{u}}_{\left(k_{0}\right)}^{+} \cos \Theta+\hat{\boldsymbol{u}}_{\left(k_{0}\right)}^{-} \sin \Theta .
$$

Then, the first-order momentum shift can be evaluated as follows: The constant spinor can be approximated as

$$
\begin{aligned}
\left(\begin{array}{c}
\cos \Omega \\
\sin \Omega
\end{array}\right) \approx & \left\{\hat{\boldsymbol{u}}_{(k)}^{+}-\left(k-k_{0}\right) \frac{\lambda_{C}}{2 \gamma_{0}^{2}} \hat{\boldsymbol{u}}_{\left(k_{0}\right)}^{-}\right\} \cos \Theta \\
& +\left\{\hat{\boldsymbol{u}}_{(k)}^{-}+\left(k-k_{0}\right) \frac{\lambda_{C}}{2 \gamma_{0}^{2}} \hat{\boldsymbol{u}}_{\left(k_{0}\right)}^{+}\right\} \sin \Theta
\end{aligned}
$$

and therefore the spinor wave packet $\Phi$ can be decomposed to pure-energy components as

$$
\begin{aligned}
\hat{f}^{+}(k)= & \left(\hat{\boldsymbol{u}}_{(k)}^{+}\right)^{T} \Phi(k) \\
\approx & C \exp \left[-\frac{\left(k-k_{0}\right)^{2}}{4 \sigma_{k}^{2}}\right]\left\{1+\tan \Theta \frac{\lambda_{C}}{2 \gamma_{0}^{2}}\left(k-k_{0}\right)\right\} \cos \Theta \\
\approx & C \exp \left[-\frac{\left(k-k_{0}\right)^{2}}{4 \sigma_{k}^{2}}\right] \exp \left[\tan \Theta \frac{\lambda_{C}}{2 \gamma_{0}^{2}}\left(k-k_{0}\right)\right] \cos \Theta \\
= & C \exp \left[-\left(\frac{\lambda_{C}}{2 \gamma_{0}^{2}} \sigma_{k} \tan \Theta\right)^{2}\right] \\
& \times \exp \left[-\frac{\left(k-k_{0}-\gamma_{0}^{-2} \lambda_{C} \sigma_{k}^{2} \tan \Theta\right)^{2}}{4 \sigma_{k}^{2}}\right] \cos \Theta \quad(\mathrm{D} 5)
\end{aligned}
$$

and

$$
\begin{aligned}
\hat{f}^{-}(k)= & \left(\hat{\boldsymbol{u}}_{(k)}^{-}\right)^{T} \Phi(k) \\
\approx & C \exp \left[-\frac{\left(k-k_{0}\right)^{2}}{4 \sigma_{k}^{2}}\right]\left\{1-\cot \Theta \frac{\lambda_{C}}{2 \gamma_{0}^{2}}\left(k-k_{0}\right)\right\} \\
& \times \sin \Theta \\
\approx & C \exp \left[-\frac{\left(k-k_{0}\right)^{2}}{4 \sigma_{k}^{2}}\right] \exp \left[-\cot \Theta \frac{\lambda_{C}}{2 \gamma_{0}^{2}}\left(k-k_{0}\right)\right] \\
& \times \sin \Theta \\
= & C \exp \left[-\left(\frac{\lambda_{C}}{2 \gamma_{0}^{2}} \sigma_{k} \cot \Theta\right)^{2}\right] \\
& \times \exp \left[-\frac{\left(k-k_{0}+\gamma_{0}^{-2} \lambda_{C} \sigma_{k}^{2} \cot \Theta\right)^{2}}{4 \sigma_{k}^{2}}\right] \sin \Theta .
\end{aligned}
$$


It is observed that $\hat{f}^{+}(k)$ is shifted by $+\gamma_{0}^{-2} \lambda_{C} \sigma_{k}^{2} \tan \Theta$ and $\hat{f}^{-}(k)$ is shifted by $-\gamma_{0}^{-2} \lambda_{C} \sigma_{k}^{2} \cot \Theta$. It is to be noted that the momentum shifts are quadratically proportional to the momentum spread. Since the velocities of an electron and a positron are given by $+\frac{p}{\gamma m}$ and $-\frac{p}{\gamma m}$, respectively, and their effective momentum shifts are $\delta p^{ \pm}= \pm(\tan \Theta)^{ \pm 1} \gamma_{0}^{-2} \hbar \lambda_{C} \sigma_{k}^{2}=$ $\pm(\tan \Theta)^{ \pm 1} \sigma_{p}^{2} / \gamma_{0}^{2} m c$, they both drift in the same direction with a velocity of $\delta v^{ \pm}=(\tan \Theta)^{ \pm 1} \sigma_{p}^{2} / \gamma_{0}^{3} m^{2} c$, respectively.
[1] P. A. M. Dirac, Proc. R. Soc. London, Ser. A 117, 610 (1928).

[2] B. Thaller, The Dirac Equation (Springer, Berlin, 1992).

[3] C. D. Anderson, Phys. Rev. 43, 491 (1933).

[4] O. Klein, Z. Phys. 53, 157 (1929).

[5] E. Schrödinger, Sitzungsber. Preuss. Akad. Wiss. Phys. Math. K1. 24, 418 (1930).

[6] J. J. Sakurai, Advanced Quantum Mechanics (Addison-Wesley, Reading, MA, 1967).

[7] D. Hestenes, Found. Phys. 20, 1213 (1990).

[8] K. Huang, Am. J. Phys. 20, 479 (1952).

[9] C. G. Darwin, Proc. R. Soc. London, Ser. A 118, 654 (1928).

[10] P. Krekora, Q. Su, and R. Grobe, Phys. Rev. Lett. 93, 043004 (2004).

[11] Z. Y. Wang and C. D. Xiong, Phys. Rev. A 77, 045402 (2008).

[12] B. G. Sidharth, Int. J. Theor. Phys. 48, 497 (2009).

[13] R. Gerritsma, G. Kirchmair, F. Zahringer, E. Solano, R. Blatt, and C. F. Roos, Nature (London) 463, 68 (2010).

[14] T. M. Rusin and W. Zawadzki, Phys. Rev. D 82, 125031 (2010).

[15] L. Lamata, J. Casanova, R. Gerritsma, C. F. Roos, J. J. GarciaRipoll, and E. Solano, New J. Phys. 13, 095003 (2011).

[16] D. W. Zhang, Z. D. Wang, and S. L. Zhu, Front. Phys. 7, 31 (2012).

[17] W. Zawadzki and T. M. Rusin, J. Phys. Condens. Matter 23, 143201 (2011).

[18] V. Y. Demikhovskii, G. M. Maksimova, A. A. Perov, and E. V. Frolova, Phys. Rev. A 82, 052115 (2010).
[19] G. Dávid and J. Cserti, Phys. Rev. B 81, 121417 (2010).

[20] D. Hestenes, Found. Phys. 40, 1 (2010).

[21] J. W. Braun, Q. Su, and R. Grobe, Phys. Rev. A 59, 604 (1999).

[22] A. Maquet and R. Grobe, J. Mod. Opt. 49, 2001 (2002).

[23] J. San Roman, L. Roso, and L. Plaja, J. Phys. B 36, 2253 (2003).

[24] E. Ackad and M. Horbatsch, J. Phys. A 38, 3157 (2005).

[25] G. R. Mocken and C. H. Keitel, Comput. Phys. Commun. 178, 868 (2008).

[26] L. L. Foldy and S. A. Wouthuysen, Phys. Rev. 78, 29 (1950).

[27] J. D. Bjorken and S. D. Drell, Relativistic Quantum Mechanics (McGraw-Hill, New York, 1964).

[28] R. F. O’Connell, Mod. Phys. Lett. A 26, 469 (2011).

[29] T. M. Rusin and W. Zawadzki, Phys. Rev. A 84, 062124 (2011).

[30] S. T. Park and A. H. Zewail, J. Phys. Chem. A 116, 11128 (2012).

[31] F. Bakke and H. Wergelan, Physica 69, 5 (1973).

[32] C. Almeida and A. Jabs, Am. J. Phys. 52, 921 (1984).

[33] See Supplemental Material at http://link.aps.org/supplemental/ 10.1103/PhysRevA.86.062105 for more computational results of Zitterbewegung and spinor states.

[34] N. Hamdan, A. Altorra, and H. A. Salman, Proc. Pak. Acad. Sci. 44, 263 (2007).

[35] B. Thaller, Advanced Visual Quantum Mechanics (Springer, New York, 2005).

[36] B. Thaller, arXiv:quant-ph/0409079.

[37] W. Greiner, Relativistic Quantum Mechanics (Springer, Berlin, 2000). 